\title{
Inhibitory effects of copper on bacterial and fungal growth
}

\author{
Khan M.A ${ }^{1}$, Yaqoob $S^{2}$ \\ ${ }^{1}$ Dr. Mohsin Ali Khan, Secretary E.E.T, ${ }^{2}$ Dr. ShadmaYaqoob, Associate Professor, both authors are attached with \\ Department of Microbiology, Era's Lucknow Medical College and Hospital, Lucknow, UP, India.
}

Address for Correspondence: Dr. Shadma Yaqoob, 20/253, Sector-20, Indra Nagar, Lucknow. Emaildrshadmayaqoob@yahoo.com

\begin{abstract}
Introduction: Researchers conducted a literature, technology and patent search that traced the history of understanding the "bacteriostatic and sanitizing properties of copper and copper alloy surfaces" which demonstrated that copper, in very small quantities, has the Copper alloy surfaces have intrinsic properties to destroy a wide range of microorganisms. Today copper, in the form of plumbing tube, copper or copper-alloy surfaces proved to be a significant step in decreasing the fungal and bacterial infections in hospitals. Aims and objective: To know the bactericidal and fungicidal properties of copper for its implication in various areas in preventing nosocomial infection. Material and Methods: Eight sterile petri dishes (four for Blood agar media and four for MacConkey agar media) and two sterile $30 \mathrm{ml}$ screw capped bottles (for Sabouraud's Dextrose agar media) were taken. Sterile copper discs were placed in four plates of Blood agar and MacConkey agar media and other four plates were without copper discs. In the same way copper piece was placed in one of the bottle with Sabourauds Dextrose agar media. Pure growths of E. coli, Klebsiella and candida were inoculated and incubated. Results: The plates with bacterial and fungal growth were reported accordingly. The growth was significantly reduced in Plates and bottles with copper discs. Conclusion: Copper alloy surfaces have intrinsic properties to destroy a wide range of microorganisms so copper's use in water supplies and surfaces are recommended.
\end{abstract}

Keywords: Copper, Bactericidal, Fungicidal, Nosocomial infection

\section{Introduction}

Metal ions, either alone or in complexes, have been used for centuries to disinfect fluids, solids and tissues $[1,2]$. The ancient Greeks of the pre-Christian era of Hypocrates (400 BC) were the first to discover the sanitizing power of copper thousands of years ago. They prescribed copper for pulmonary diseases and for purifying drinking water. The oldest recorded medical use of copper is mentioned in the Smith Papyrus, one of the oldest books known. Egyptian medical text, written between 2600 and 2200 B.C., describes the application of copper to sterilize chest wounds and drinking water. The use of copper in medicine became widespread in the $19^{\text {th }}$ and early $20^{\text {th }}$ century [3]. Metals such as silver, iron, and copper could be used for environmental control, disinfection of water, or reusable medical devices or incorporated into medical devices (e.g., intravascular catheters) [4]. The bactericidal, fungicidal

Manuscript received: $4^{\text {th }}$ April 2017

Reviewed: $14^{\text {th }}$ April 2017

Author Corrected: $22^{\text {nd }}$ April 2017

Accepted for Publication: $29^{\text {th }}$ April 2017 and, to some extent, virucidal properties of copper, copper compounds and alloys of copper have been known for many years. There is evidence indicating "bacteriostatic and sanitizing properties of copper and copper alloy surfaces". Certain studies shows that the copper tubing used for in-hospital water transport and treatment systems may help to reduce the numbers of undesirable bacteria. Similarly, the idea of using copper vessels to render water drinkable has been revived only very recently as a low-cost alternative for developing countries [5]. Currently, there is an intense interest in the use of copper as a self-sanitizing material,

Mechanisms of antibacterial action of copper- The antimicrobial properties of copper are still under active investigation. Molecular mechanisms responsible for the antibacterial action of copper have been a subject of intensive research. Scientists are also actively demonstrating the intrinsic efficacies of copper alloy touch surfaces to destroy a wide range of 
microorganisms that threaten public health. In 1973, researchers at Battelle Columbus Laboratories conducted a comprehensive literature, technology and patent search that traced the history of understanding the "bacteriostatic and sanitizing properties of copper and copper alloy surfaces" which demonstrated that copper, in very small quantities, has the power to control a wide range of molds, fungi, algae and harmful microbes [6].

Numerous papers are there with different investigations showing copper's antimicrobial mechanisms and efficacy of copper's action on microbes. The authors noted that the antimicrobial mechanisms are very complex and take place in many ways, both inside cells and in the interstitial spaces between cells. Some of the molecular mechanisms mentioned by researchers are:

- The copper altered the 3-dimensional structure of proteins and disrupt the enzyme structures resulting in inactivation of bacteria or viruses $[7,8]$.

- Copper produces deleterious effects in superoxide radicals, generating $\mathrm{OH}$ - radicals, thereby causing "multiple hit damage" at target sites [9].

- Copper with lipids produces holes in the cell membranes damaging the integrity of cell and leaking of essential nutrients leading to cell deaths $[10,11]$

Many recent publications also showed that microorganisms are rapidly killed on metallic copper surfaces by 'contact killing' mechanism [12].

Copper alloy surfaces have intrinsic properties to destroy a wide range of microorganisms. In the interest of protecting public health, especially in healthcare environments many studies have been conducted in the past ten years regarding copper's efficacy to destroy microorganisms. The bactericidal, fungicidal and, to some extent, virucidal properties of copper, copper compounds and alloys of copper have been known for many years. Indeed, the earliest medical texts refer to the use of copper compounds for wound-healing, i.e., sterilization of wounds [3]. There is evidence indicating that copper tubing used for in-hospital water transport and treatment systems may help to reduce the numbers of undesirable bacteria present in water. Today, copper, in the form of plumbing tube, copper or copper-alloy surfaces, has shown it is a significant step to the contraction of fungal and bacterial disease in healthcare facilities. The three major areas where copper has been used to stem nosocomial infections include the sanitation of the water supply, of air-conditioning systems, and of surfaces. The CDC addresses copper's use in two of these, water supplies and surfaces, in its draft guidelines [13].

\section{Material \& Methods}

This study was conducted after approval from institutional ethical committee in the Department of Microbiology, for a period of one year, from February 2014 to April 2015.

\section{Study Design: Observational Study}

Setting: Eras Lucknow medical university, Microbiology department

Specimens and instrument required

- Pure growth of E.coli and Klebsiella species(sp.), Candida sp.

- Copper discs of approx $90 \mathrm{~mm}$ (petri dish size).

- Copper pieces of size $2 * 1 \mathrm{~cm}$.

- Blood agar plate, MacConkey agar plate, Sabourauds Dextrose agar media.

\section{Methods}

Part I (media preparation).

- Eight sterile petri dishes (four for Blood agar media and four for MacConkey agar media) and two sterile $30 \mathrm{ml}$ screw capped bottles (for Sabouraud's Dextrose agar media) were taken.

- Put sterile copper discs in four petri dishes.

- The melted sterile Blood agar media were poured in two petri dishes ie: one with copper discs and one without copper discs. MacConkey agar media was also poured in same pattern.

- Dishes were left undisturbed until the medium was set.

- In the same way sabourauds Dextrose agar media was poured in two bottles (one with copper piece and one without).

- Bottles were kept in tilted position so that slant was formed and left undisturbed until the medium was set. 


\section{Original Research Article}

Part II (culture)

- Two to three colonies from Pure growths of E.coli and Klebsiella were taken and emulsified in peptone water. This inoculum was kept for 30 minutes.

- After 30 minutes, the inoculum was inoculated on two sets of plates as prepared above.
- $\quad$ All the inoculated plates were incubated at $37^{0} \mathrm{C}$ for 24 hours.

- Pure growth of Candida species was inoculated on two Sabourauds Dextrose agar media in bottles (one with copper piece and one without copper) and kept at $27^{\circ} \mathrm{C}$ for one week.

\section{Observation / Result}

The study was conducted in the Microbiology department of Era's Lucknow Medical college and Hospitals during Feb 2014 to April 2015. Pure growth of E.coli, Klebsiella, Candida were cultured and screened for growth on Blood agar, MacConkey agar and Sabourauds Dextrose agar media with and without copper.

\section{Growth of E.coli [figure 1, figure 2].}

\begin{tabular}{|c|c|c|}
\hline Media & Without copper discs & With copper discs \\
\hline Blood agar & Heavy growth & Less growth \\
\hline MacConkey agar & Heavy growth & No growth \\
\hline
\end{tabular}

Table 1 showing growth of E. coli on Blood agar and MacConkey agar with and without copper discs were compared. Growth was significantly reduced on Blood agar plates having copper discs and was almost nil on MacConkey agar plates with copper.

Figures showing inhibitory effects of copper on bacterial and fungal growth.

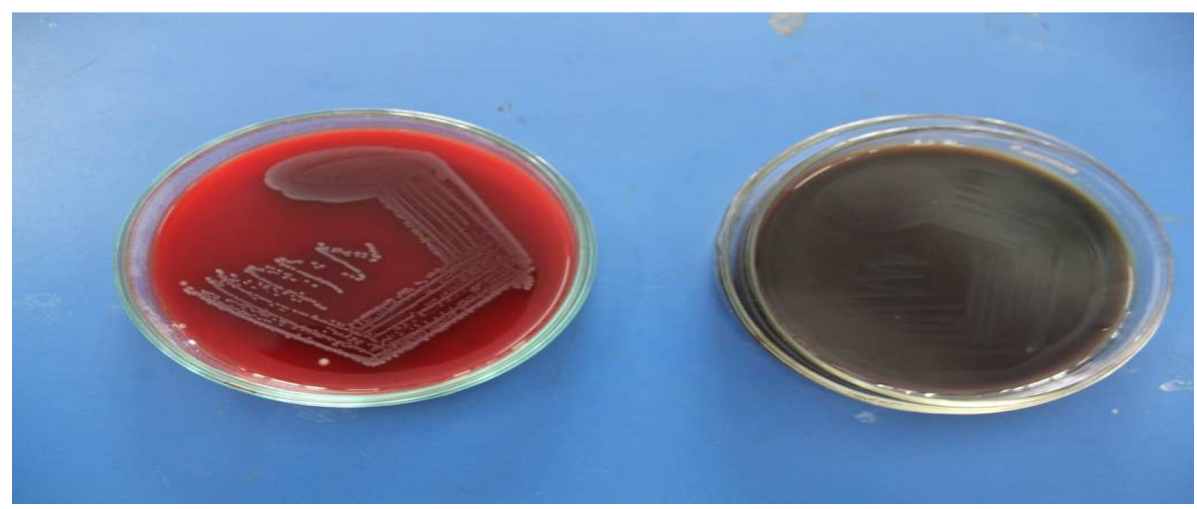

Figure-1: reduced growth of E coli on Blood agar with copper disc

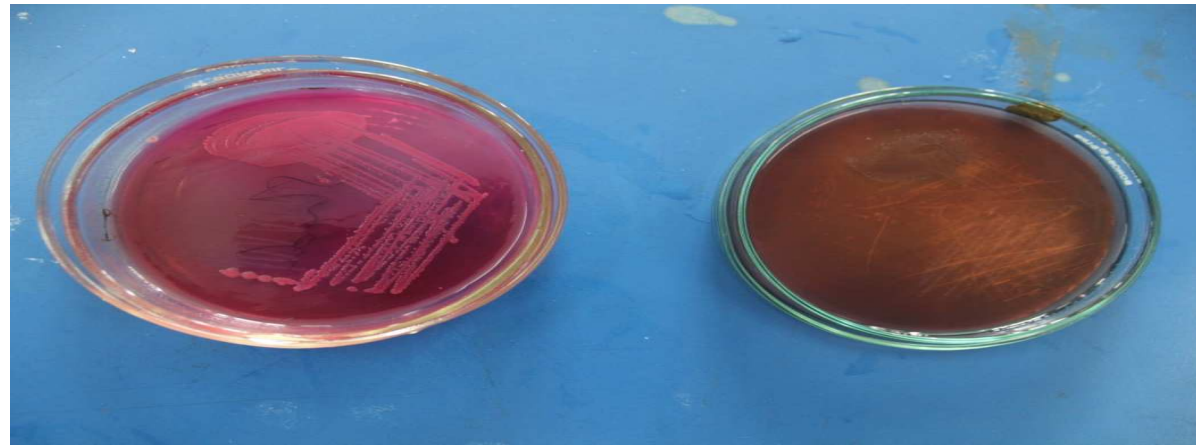

Figure-2: reduced growth of E coli on MacConkey agar with copper disc 


\section{Growth of Klebsiella [figure 3].}

\begin{tabular}{|c|c|c|}
\hline Media & Without copper discs & With copper discs \\
\hline Blood agar & Heavy growth & Less growth \\
\hline MacConkey agar & Heavy growth & Less growth \\
\hline
\end{tabular}

Table 2 showing the growth of Klebsiella sp on Blood agar and MacConkey agar with and without copper discs. Growth was significantly reduced on both Blood agar and MacConkey agar plates with copper.

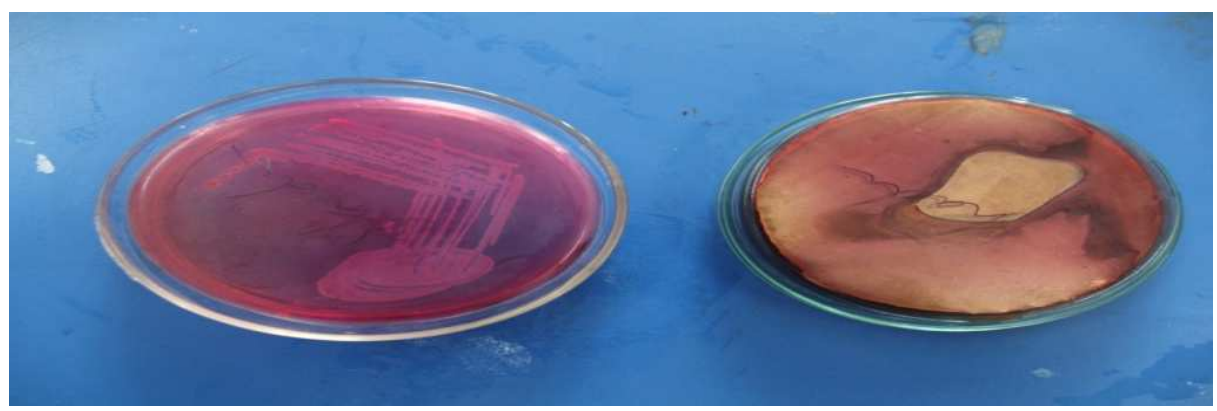

Figure-3: Reduced growth of Klebsiella on MacConkey Agar Plate with Copper discs

\section{Growth of Candida [figure 4]}

\begin{tabular}{|c|c|c|}
\hline Media & Without copper piece & With copper piece \\
\hline Sabourauds Dextrose Agar & Heavy growth & Less growth \\
\hline
\end{tabular}

Table 3 showing that the growth of candida sp on Sabourauds Dextrose agar with copper piece was significantly reduced.

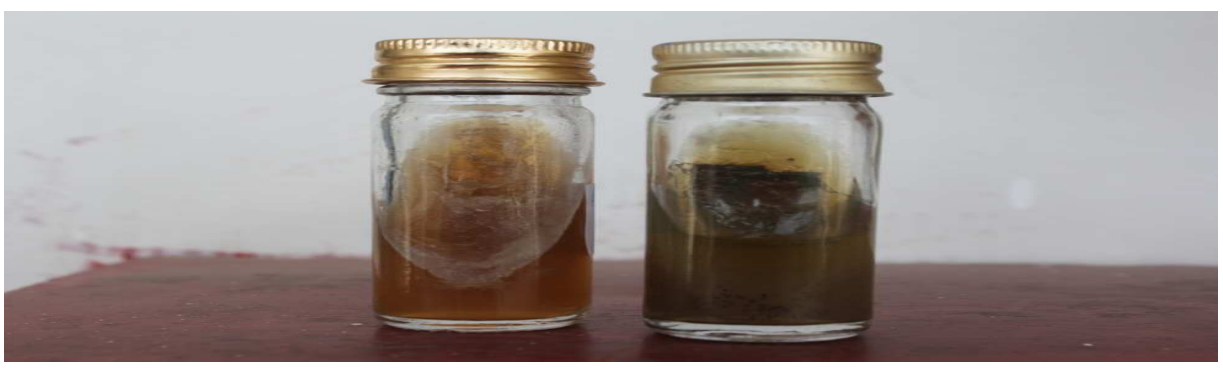

Figure-4: Reduced growth of Candida species on SDA with copper piece.

\section{Discussion}

Copper surfaces, with their self-sanitizing properties, could be envisioned as making an important contribution to infection control. Thus, the use of antimicrobial metallic copper surfaces is likely to provide protection from infectious microbes by reducing surface contamination, as recently shown in successful hospital trials. Hospital trials are now ongoing worldwide $[14,15]$. Data in the study by Christopher showed that dry metallic copper surfaces rapidly and efficiently kill bacteria [16].

The bacteriostatic effect of copper was also noted by Dr. Phyllis J. Kuhn, who was involved in the training of
House keeping and maintenance personnel in the Hamot Medical Center, Pennsylvania. She investigated bacterial growth on metals. Small strips of stainless steel, brass, aluminum, and copper, were inoculated with broths of Escherichia coli, Staphylococcus aureus, and Pseudomonas species. The copper and brass strips showed little or no growth, while the aluminum and stainless steel strips produced a heavy growth of all microbes [17].

This study was very much similar to our study in which less growth of E.coli was present on plates containing copper. 
Recent studies have shown that copper alloy surfaces kill E. coli O157:H7 [18,19,20]. In one of the study, E. coli O157:H7 was rapidly and almost completely killed within ninety minutes at room temperature on an alloy containing $99.9 \%$ copper. In our study also, we observed the killing of E.coli and Klebsiella sp on culture media having copper. After $24 \mathrm{hrs}$ of incubation the growth of the bacteria was significantly reduced on the Blood agar and MacConkey agar plates containing copper discs. Another study conducted with E.coli but with different way by The Midwest Research Institute, USA showing the similar results of reduction of the E.coli growth on copper surfaces. In this study bacteria were introduced into fifty foot coils of different plumbing tube materials. Water with a suspension of E. coli pumped through the coils. In different types of plumbing material, including glass, the level of bacteria remained the same or increased but in the copper loop only $1 \%$ of the E. coli bacteria remained viable after five hours. Our results also showed the antimicrobial property of copper but the method was different [21].

In one of the study the antifungal efficacy of copper was compared to aluminium on the following organisms that can cause human infections: Aspergillus spp., Fusariumspp., Penicilliumc, Aspergillus niger and Candida albicans [22]. An increased die-off of fungal growth was found on copper surfaces. We also did experiments with Candida albicans. The growth was significantly reduced in sabourauds Dextrose agar media with copper piece and heavy growth was present on bottle with no copper piece.

\section{Conclusion}

The antimicrobial properties of copper surfaces have now been firmly established. Hospital trials have shown a reduction in bacterial counts, indicating that copper surfaces are really additional tool along with other hygienic measures to decrease the number and severity of nosocomial infections. Additional studies should be done in determining the most cost-effective way for the protection of hospitals so that different sites like doorknobs, bed rails, plumbing lines, working surfaces should be made of copper.

This simple experiment in the laboratory proved the bactericidal and fungicidal properties of copper. So the copper and its alloys can be implicated in various areas in the hospital as one of the hygienic measures thus helping in prevention of nosocomial infection.
Acknowledgement- We thank the staff for their assistance to carry out this study.

\section{Funding: Nil, Conflict of interest: None Permission of IRB: Yes}

\section{References}

1. Airey P, Verran J. Potential use of copper as a hygienic surface. problems associated with cumulative soiling and cleaning. J. Hosp. Infect. 2007;67(3):272278. Doi10.1016/j.jhin.2007.09.002

2. Block SS. Disinfection,Sterilisation and Preservation. 2001; 9:1857.

3. Dollwet HHA, Sorenson JRJ. Historic uses of copper compounds in medicine.Trace Elements in Medicine. $1985 ; 2.2: 80-87$.

4. Weber DJ, Rutala WA. Use of metals as microbicides in preventing infections in healthcare. Philadelphia: Lippincott Williams \& Wilkins .2001:415-30.

5. Sudha VB, Singh KO, Prasad SR, Venkatasubramanian P. Killing of enteric bacteria in drinking water by a copper device for use in the home: laboratory evidence. Trans R Soc Trop Med Hyg. 2009 Aug; 103(8):819-22. doi: 10.1016/j.trstmh.2009.01.019. Epub 2009 Feb 23.

6. Dick RJ, Wray JA, Johnston HN. A Literature and Technology Search on the Bacteriostatic and Sanitizing Properties of Copper and Copper Alloy Surfaces. Phase 1 Final Report, INCRA Project 212, June 29, 1973; contracted to Battelle Columbus Laboratories, Columbus, Ohio

7. Thurman RB, Gerba CP.The Molecular Mechanisms of Copper and Silver Ion Disinfection of Bacteria and Viruses. CRC Critical Reviews in Environmental Control.1989;18(4):295-315. Doi:10.1080/ 10643388 909388351 .

8. Sterritt RM, Lester JN. Interactions of heavy metals with bacteria. Sci Total Environ. 1980 Jan; 14(1): 5-17.

9. Samuni A, Aronovitch J, Godinger D, Chevion M, Czapski G. On the cytotoxicity of vitamin $\mathrm{C}$ and metal ions. A site-specific Fenton mechanism. Eur J Biochem. 1983 Dec 1;137(1-2):119-24. 
10. Samuni A, Chevion M, Czapski G. Roles of Copper and Superoxide Anion Radicals in the Radiation-Induced Inactivation of T7 Bacteriophage. Radiat. Res. JSTOR 1984; 99(3):562-572.

11. Manzl C, Enrich J, Ebner H, Dallinger R, Krumschnabel G. Copper-induced formation of reactive oxygen species causes cell death and disruption of calcium homeostasis in trout hepatocytes. Journal of Toxicology.2004; 196.(1-2): 57-64. Doi:10.1016/j.tox. 2003. 11.001 .

12. Gregor G, Christopher R, Marc S. Metallic Copper as an Antimicrobial SurfaceAppl. Environ. Microbiol. March 2011 vol. 77 ( 5):1541-1547. doi: 10.1128/AEM. 02766-10

13. Centers for Disease Control and Prevention. Dept. of Health and Human Services.www.cdc.gov.

14. Casey AL, Adams D, Karpanen TJ, Lambert PA, Cookson BD, Nightingale P, Miruszenko L, Shillam R, Christian P, Elliott TS. Role of copper in reducing hospital environment contamination. J Hosp Infect. 2010 Jan; 74(1):72-7. doi: 10.1016/j.jhin.2009.08.018. Epub 2009 Nov 20.

15. Marais F, Mehtar S, Chalkley L. Antimicrobial efficacy of copper touch surfaces in reducing environmental bioburden in a South African community healthcare facility. J Hosp Infect. 2010 Jan;74(1):80-2. doi: 10.1016/j.jhin.2009.07.010. Epub 2009 Sep 25.

16. Christophe E S, Christian G E, Davide Q, Dylan W D, Christopher J C, and Gregor G. Bacterial Killing by
Dry Metallic Copper Surfaces. Appl. Environ. Microbiol. 2011; 77(3): 794-802.doi.10.1128/ AEM. 01599-10.

17. Kuhn PJ. Doorknobs: a source of nosocomial infection. Diagnost Med 1983:62e63.

18. Michels HT, Wilks SA, Noyce JO, Keevil C.W. Copper Alloys for Human Infectious Disease Control. Presented at Materials Science and Technology Conference, September 2005; 25-28, Pittsburgh, PA. Copper for the 21st Century Symposium.

19. Wilks SA, Michels H, Keevil CW. The survival of Escherichia coli $\mathrm{O} 157$ on a range of metal surfaces. International Journal of Food Microbiology .2005;105(3):445-54. Doi.10.1016/j. ijfoodmicro. 2005. 04.021 .

20. Espírito Santo C, Taudte N, Nies DH, Grass G. Contribution of copper ion resistance to survival of Escherichia coli on metallic copper surfaces. Appl Environ Microbiol. 2008 Feb;74(4):977-86. Epub 2007 Dec 21.

21. Wells, F., Midwest Res Inst 2001, 348 (348C/348D), 48.

22. Weaver L, Michels HT, Keevil CW. Potential for preventing spread of fungi in air-conditioning systems constructed using copper instead of aluminium.Lett Appl Microbiol. 2010 Jan;50(1):18-23. doi: 10.1111/j. 1472-765X.2009.02753.x.

\section{How to cite this article?}

Khan M.A, Yaqoob S. Inhibitory effects of copper on bacterial and fungal growth. Int J Med Res Rev 2017;5(05):466471. doi:10.17511/ijmrr. 2017.i05.05. 